\title{
7
}

\section{Left Ventricle Postinfarction Aneurism: Comparison Between Diagnostic Value of Different Methods of Visualization}

\author{
Maryna N. Dolzhenko, Sergey V. Potashev and Natalia N. Nosenko \\ The P.L.Shupik National Medical Academy Of Postgraduate Education/Cardiology \\ And Functional Diagnosis Department \\ Ukraine
}

\section{Introduction}

Today method of coronary angiography and ventriculography (CVG) is a "golden standard" in diagnosis of coronary arteries atherosclerosis (Masaki Y. et al., 2005). Besides, CVG is successfully used for left ventricle (LV) global contractility evaluation and LV aneurism diagnosis in the patients after acute myocardial infarction (AMI) before surgeon revascularization and decision about LV surgeon plastics. But being invasive, this method has certain limitations and cannot be suitable for dynamic medical supervision afterwards (Schuijf J.D. et al., 2004). It should be mentioned, that possibility of coronary atherosclerosis diagnosis is not necessarily connected with arterial lumen narrowing. Atherosclerotic plague prolapses into arterial lumen, causing its narrowing and relevant clinical symptoms. But early stages of atherosclerosis usually do not lead to hemodynamically significant patency decrease and may not be seen by means of CVG. That is why design of new methods of coronary pathology, especially non-invasive, is of great importance. Among the demands to up-to-date examination methods high specificity, sensitivity, accuracy and safety should be mentioned, as well as high repeatability and economical suitability. All these features are attributes of multislice spiral computed tomography (MSCT) (Laissy et al., 2007; Masaki et al., 2005), what explains constantly growing interest to this method. Main advantage of Doppler echocardiography (EchoCG) is that it allows non-invasively and in real time to evaluate dimensions and behaviour of cardiac structures, to obtain characteristics of heart hemodynamics and indirect impression of chamber and main vessels pressures. Significant comparability of EchoCG results with chambers catheterization data has been long proven (Becher et al., 2004; Swedberg et al, 2005). Postinfarction LV aneurism promotes LV remodeling, worsens its systolic and diastolic function. LV myocardial function may improve in case of adequate vascularization revival due to surgeon procedure, for instance coronary arteries bypass graft (CABG) (Becher et al., 2004; Budoff, 2004; Dirksen et al., 2002; Dolzhenko et al.. 2007). Aneurism resection significantly influences LV remodelling, promotes chamber pressures decrease and slows down heart failure (HF) progression (Dolzhenko et al., 2008). That is why cardiologist and cardiac surgeon need maximum of objective information not only about coronary arteries patency, but also about LV structural and functional changes in order to adequately evaluate severity of operational 
risk and long-term postoperational prognosis (Budoff, 2004; Dirksen et al., 2002; Dolzhenko et al., 2008). Up to now there were few attempts to compare informative diagnostic value of invasive and non-invasive methods of LV structural anomalies in the patients after AMI.

Among those, there are promising data regarding high correlation $(r=0.76)$ of non-invasive evaluation of left ventricle (LV) ejection fraction (EF) between resting echocardiographic EFs and single photon emission computed tomography (SPECT) resting gated sestamibi images in patients with single-vessel disease, and a moderate correlation $(r=0.68$ and $r=0.68)$ in patients with 2- and 3-vessel disease, respectively, while patients with two and 3-vessel disease were statistically more likely to have RWMAs detected by gated SPECT sestamibi than by echo (Fleming, 2002).

It has also been shown, that changes in resting LVEF and high-dose dipyridamole pharmacologically induced stress LVEF (SEF) provide a valuable diagnostic marker as to the number of significantly diseased coronary arteries and can be acquired from gated SPECT sestamibi images (Fleming\&Boyd, 2002).

The aim of this study was to compare the efficacy of modern methods of heart left chambers visualization in the patients after acute myocardial infarction (AMI) with LV aneurism before coronary arteries bypass graft (CABG) combined with LV aneurismectomy $(\mathrm{CABG}+\mathrm{AE})$ in LV global contractility evaluation and reliability in LV chronic aneurism and its thrombosis diagnosis.

\section{Methods}

The study was approved by local ethics committee.

In the study we prospectively included 116 patients after AMI with LV postinfarction aneurism (LVA) without significant valvular dysfunction eligible for CABG combined with LVA resection. Exclusion criteria were a history of recent myocardial infarction (4 weeks before pre-operative angiography), atrial fibrillation, significant valvular heart disease or previous $\mathrm{CABG}$. During and after the $\mathrm{CABG}$, standard laboratory markers for myocardial infarction were obtained and none of the patients was diagnosed with perioperative myocardial infarction. Medication treatment in all the post-infarction patients included aspirin, statin, beta-blocker, ACE inhibitor and nitrates, if indicated. All patients underwent EchoCG and MSCT prior to the operation. Fourty age-matched subjects with CAD and without AMI history, who underwent CVG, EchoCG and contrast MSCT for coronary revascularization decision, served as controls. Program of the study included X-ray contrast CVG, MSCT with chambers contrast and Doppler EchoCG.

\subsection{Coronary angiography}

Coronary angiography with ventriculography was conducted and interpreted by trained physicians 1 week preceding $\mathrm{CABG}+\mathrm{AE}$. A $50 \%$ or more reduction of the luminal diameter in 2 orthogonal projections of a major coronary artery or one of its major branches or a bypass graft was considered to be significant for CAD. It is known, that LV postinfarction aneurism is a transmural scar with typical smooth inner surface without trabecular structures. LV wall is usually very thin in this place, causing inner and outer wall surfaces bulging. During systole the involved LV segment are akynetic or dyskinetic (showing paradox bulging movement) (Fleisher et al., 2007). LV aneurism and its thrombosis diagnosis during CVG with further confirmation ad oculus during operation was used as 
diagnostic "golden standard" (Fleisher et al., 2007). LV global contractility was evaluated by ejection fraction (EF) calculation by Simpson disc method in right anterior oblique 300 projection (Fleisher et al., 2007; Scanlon et al., 1999).

\subsection{Echocardiography}

A standard clinical echocardiographer, equipped with pulsed-wave TDI option (Medison "SonoAce" 9900) was used. Recordings and calculations of different parameters, including $\mathrm{LV}$ chamber volumes and $\mathrm{EF}$, were performed according to the recommendations of the American Society of Echocardiography(Scanlon et al., 1999). LV global contractility was evaluated by by Simpson disc method in 4- and 2-chamber apical positions by calculating end-diastolic (EDV, $\mathrm{ml}$ ) and end-systolic (ESV, $\mathrm{ml}$ ) volumes with their indices to body surface area (EDI and ESI, $\mathrm{ml} / \mathrm{m}^{2}$ ) and $\mathrm{LV} \mathrm{EF,} \mathrm{\% .} \mathrm{LV} \mathrm{aneurism} \mathrm{was} \mathrm{defined} \mathrm{as} \mathrm{transmural} \mathrm{a-}$ or dyskinetic scar tissue with distinct smooth inner surface involving two or more LV segments (Dolzhenko et al., 2008; Fleisher et al., 2007).

\subsection{Multislice computed tomography}

MSCT was performed on tomographer «Light Speed-16» ("General Electric Company», USA) using cardiological «Advantage Workstation 4.2» («General Electric Company», USA). Spiral mode of tomography with $2,5 \mathrm{~mm}$ thick slice and retrospective ECG synchronization was intravenous ed with 6-8 seconds scanning time and $360^{\circ}$ rotation. Study was performed at breath held after infusomat "Omnipac" intravenous infusion. Exposure dose constituted $2,2 \mathrm{mSv}$ per one study at 16 slices per 200 frames. LV global contractility was evaluated by LV EF calculation by Simpson disc method in 4-chamber projection. LV aneurism was defined as transmural a- or dyskinetic fibrous scar tissue with distinct smooth inner surface without trabecular structures involving two or more LV segments (Dirksen et al., 2002; Swedberg et al., 2005).

\subsection{Statistics}

Comparison of different methods was performed using multiple regression analysis with 95\% confidence interval and correlation analysis. In comparison of diagnostic value of the studied methods we evaluated the following characteristics: accuracy (diagnostic efficacy) percentage of correct test results out of general quantity of both positive and negative results; sensitivity (Se) - percentage of subjects with positive test results in the population with the studied pathology; specificity $(\mathrm{Sp})$ - percentage of subjects with negative test results in the population with the studied pathology; positive predictive value $(+\mathrm{PV})$ probability of symptom or disease in case of positive test result; negative predictive value (PV) - case of negative (normal) test result.

The above numbered indices were calculated by formulas:

$$
\begin{aligned}
& \mathrm{Se}=\mathrm{N}(\mathrm{TP}) /(\mathrm{N}(\mathrm{TP})+\mathrm{N}(\mathrm{FN})) \times 100 \% \\
& \mathrm{Sp}=\mathrm{N}(\mathrm{TN}) /(\mathrm{N}(\mathrm{TN})+\mathrm{N}(\mathrm{FP}) \times 100 \% \\
& +\mathrm{PV}=\mathrm{N}(\mathrm{TP}) /(\mathrm{N}(\mathrm{TP})+\mathrm{N}(\mathrm{FP}) \times 100 \% \\
& -\mathrm{PV}=(\mathrm{TN}) /(\mathrm{N}(\mathrm{TN})+\mathrm{N}(\mathrm{FN}) \times 100 \%
\end{aligned}
$$


where $\mathrm{N}$ is the quantity of studied patients; TP - truly positive diagnosis; FP - false positive diagnosis; TN - truly negative diagnosis; FN - false negative diagnosis 12,13 . The results are expressed as the mean and 1 standard deviation. The parameters of patients and healthy subjects were compared using an unpaired t-test. A paired t-test was used to compare results within the same group. A P-value of $<0,05$ was considered significant.

\section{Results}

The main clinical features of the study group patients are presented in Table 1.

\begin{tabular}{|c|c|c|}
\hline Index & Abs. & $\%$ \\
\hline LV EF (\%) & $37,1 \pm 12,4$ & - \\
\hline LV EDI (ml/m²) & $112,4 \pm 28,2$ & - \\
\hline LV ESI $\left(\mathrm{ml} / \mathrm{m}^{2}\right)$ & $73,8 \pm 27,6$ & - \\
\hline Diabetes mellitus (n) & 14 & $12,1 \%$ \\
\hline Hypertension (n) & 75 & $64,7 \%$ \\
\hline Angina pectoris & 107 & $92,3 \%$ \\
\hline Functional class I & 15 & $12,9 \%$ \\
\hline Functional class II & 23 & $19,8 \%$ \\
\hline Functional class III & 64 & $55,2 \%$ \\
\hline Functional class IV & 14 & $12,1 \%$ \\
\hline \multicolumn{3}{|l|}{ Heart failure (NYHA functional class) } \\
\hline I (LV > 45\%) & 17 & $14,7 \%$ \\
\hline II $(\mathrm{LV}<45 \%)$ & 86 & $74,1 \%$ \\
\hline $\mathrm{I}(\mathrm{LV}<45 \%)$ & 13 & $11,2 \%$ \\
\hline \multicolumn{3}{|l|}{ Lesions localization } \\
\hline 3-vessels disease and/or left main (n) & 41 & $35,3 \%$ \\
\hline 2-vessels disease (n) & 43 & $37,1 \%$ \\
\hline 1 -vessel disease (n) & 32 & $27,6 \%$ \\
\hline \multicolumn{3}{|l|}{ Aneurism localization } \\
\hline Predominantly anterior LV aneurism (n) & 28 & $24,1 \%$ \\
\hline Anterior + apical aneurism + inferior $(n)$ & 38 & $32,8 \%$ \\
\hline Anterior + septal + apical aneurism (n) & 50 & $43,1 \%$ \\
\hline
\end{tabular}

Table 1. Clinical features of the patients studied 
As it is seen from the table, according to CVG data there were predominantly patients with 2- and 3-vessels disease of left main coronary artery lesion. Aneurisms according to CVG, MSCT and EchoCG data were mainly located in the anterior LV segments with frequent propagation to interventricular septum (IVS) and LV apex (43,1\% cases) and LV apex with propagation to inferior apical segments (32,8\% cases).

At comparison of results of LV global contractility (LV EF) data of different visualization methods significantly correlated between each other. EchoCG data highly correlated both with CVG $(\mathrm{r}=0,80)$ and MSCT $(\mathrm{r}=0,71, \mathrm{p}<0,0001)$. Comparison of LV EF results according to CVG and MSCT also showed good correlation ( $\mathrm{r}=0,73, \mathrm{p}<0,0001$ compared to EchoCG data) (Fig. 1, 2.). Thus, in LV global contractility evaluation all three methods were equally precise, while EchoCG is the method of preference, as having the least limitations, which corresponds to existing guidelines (Scanlon et al., 1999).

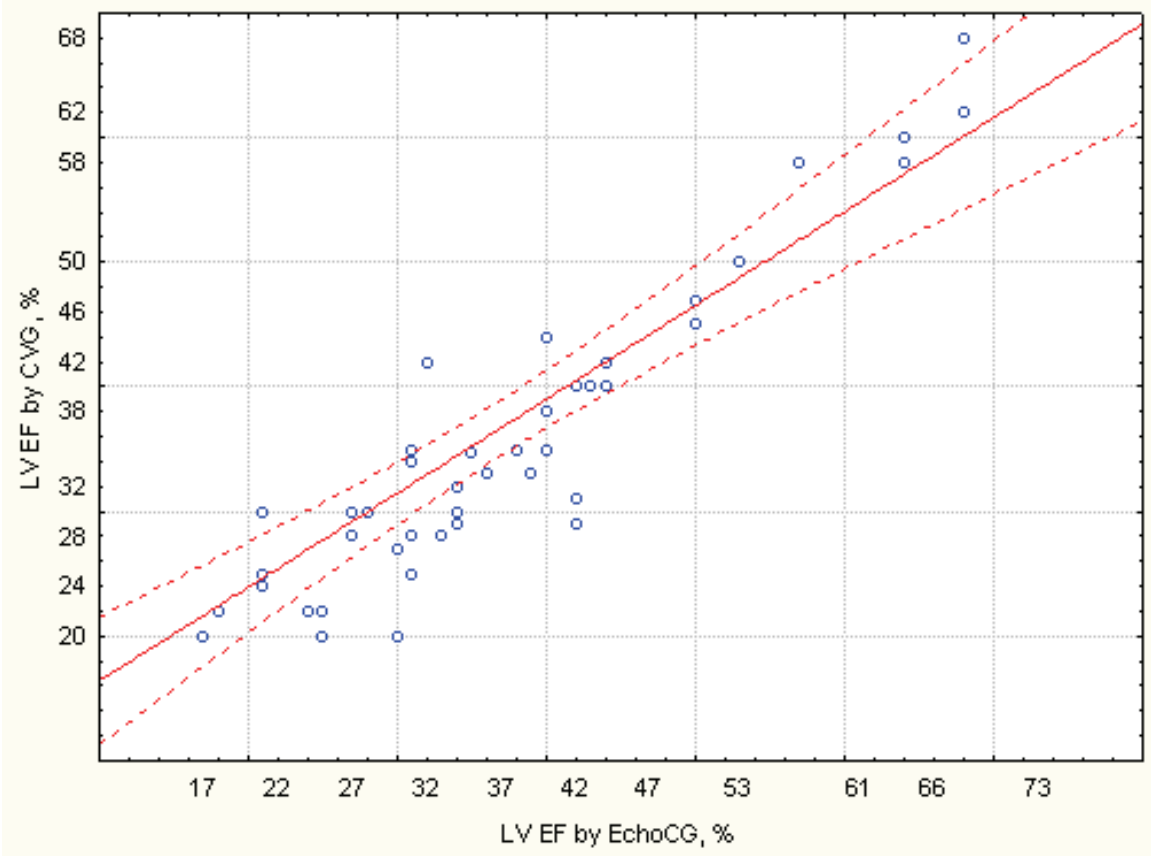

Fig. 1. Correlation of LV EF calculation according to CVG and EchoCG

At LV chronic aneurism diagnosis MSCT was the most sensitive method, which allowed diagnosis of aneurism in $100 \%$ cases. During EchoCG false negative result was obtained in 1 $(0,09 \%)$ case, but there were no false positive results. At CVG there were 7 false negative $(6,0 \%)$ cases but no false positive cases (accuracy $-86,2 \%, p<0,0001$ compared both to MSCT and EchoCG).

MSCT sensitivity in aneurism diagnosis compared to CVG was 90,8\%, while EchoCG sensitivity equaled $89,5 \%$ ( $p=0,74$ ), while methods' specificity constituted $60 \%$ and $65 \%$, respectively $(p=0,43)$. Positive predictive value of methods was $89,6 \%$ and $90,7 \%(p=0,78)$, while negative predictive value was $63,2 \%$ and $61,9 \%(p=0,84)$, respectively (Table 2$)$. 


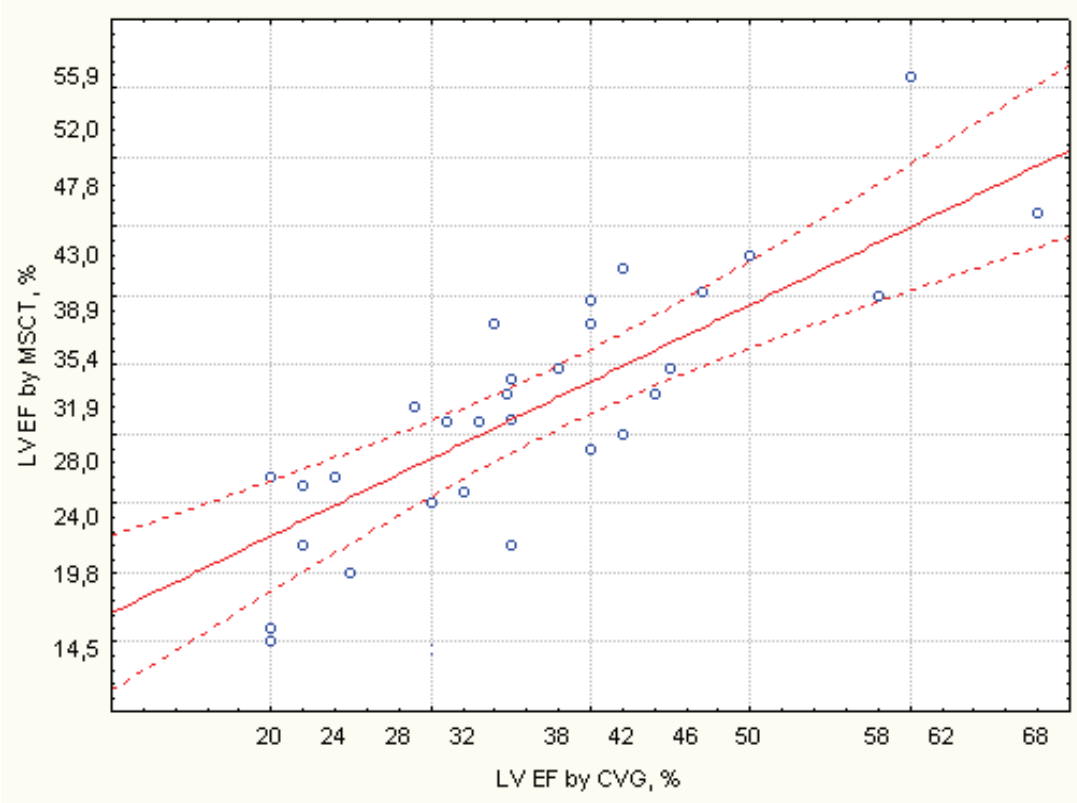

Fig. 2. Correlation of LV EF calculation according to CVG and MSCT

\begin{tabular}{|l|l|l|}
\hline MSCT & EchoCG & $p$ \\
\hline Se $=90,8 \%$ & Se $=89,5 \%$ & 0,74 \\
\hline Sp $=60,0 \%$ & Sp $=65,0 \%$ & 0,43 \\
\hline$+P V=89,6 \%$ & $+P V=90,7 \%$ & 0,78 \\
\hline$-P V=63,2 \%$ & $-P V=61,9 \%$ & 0,84 \\
\hline
\end{tabular}

Table 2. Comparison of diagnostic value of the studied methods in LV chronic aneurism diagnosis compared to CVG and post operation results

Frequency of aneurism diagnosis according to CVG data significantly correlated both with $\operatorname{MSCT}(r=0,62, p<0,0001)$ and EchoCG $(r=0,63, p<0,0001)$.

Aneurism thrombosis during operation was found in 37 (31,9\%) cases. Before operation according to CVG there were $14(12,1 \%)$ false negative and $8(6,9 \%)$ false positive aneurism thrombosis cases (accuracy $-81,0 \%$ of post operation findings). According to EchoCG data there were $5(4,3 \%)$ false negative and $2(1,7 \%)$ false positive cases (accuracy $-94,0 \%$, $\mathrm{p}=0,0031$ compared to CVG). At MSCT exams there were no false negative or false positive results (accuracy - 100\%, p<0,0001 compared to CVG). Frequency of simultaneous correct LV aneurism thrombosis diagnosis was $48,7 \%$ (18 cases).

Data regarding diagnostic value of MSCT and EchoCG compared to CVG is presented in Table 3. 


\begin{tabular}{|c|c|c|}
\hline MSCT & EchoCG & $p$ \\
\hline Se $=69,2 \%$ & Se $=61,5 \%$ & 0,22 \\
\hline $\mathrm{Sp}=79,7 \%$ & $\mathrm{Sp}=76,8 \%$ & 0,59 \\
\hline$+\mathrm{PV}=56,3 \%$ & $+\mathrm{PV}=50,0 \%$ & 0,34 \\
\hline$-\mathrm{PV}=87,3 \%$ & $-\mathrm{PV}=84,1 \%$ & 0,49 \\
\hline
\end{tabular}

Table 3. Comparison of diagnostic value of the studied methods in LV aneurism thrombosis diagnosis compared to CVG and post operation results

Frequency of LV aneurism thrombosis diagnosis by CVG correlated both with MSCT $(r=0,52, p<0,0001)$ and EchoCG $(r=0,36, p<0,0001)$. Still, the highest correlation was found between LV aneurism thrombosis diagnosis provided by MSCT and EchoCG ( $r=0,86$, $\mathrm{p}<0,0001$ compared to CVG in both cases) (Fig. 3).

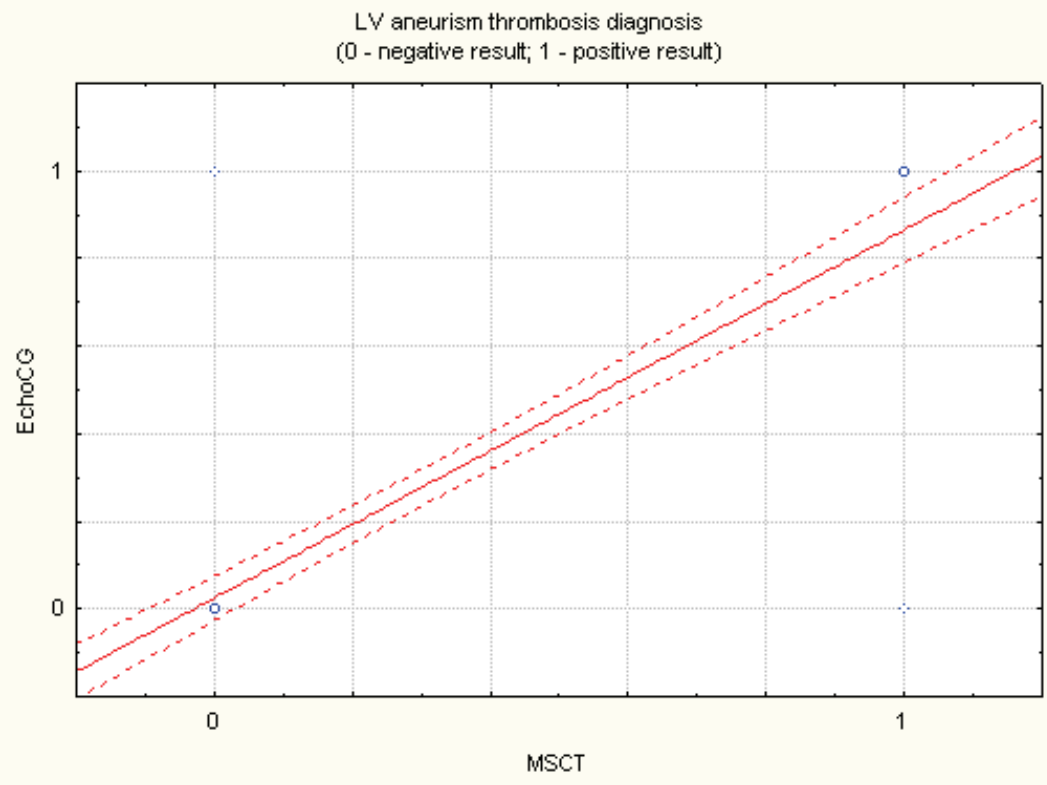

Fig. 3. Correlation of LV aneurism thrombosis diagnosis according to MSCT and EchoCG data

\section{Discussion}

According to the results of our study, MSCT showed the highest accuracy in diagnosis of LV chronic aneurism, as well as its thrombosis, compared to CVG and EchoCG. MSCT and EchoCG also showed high sensitivity and positive predictive value in diagnosis of LV chronic aneurism and high specificity and negative predictive value in diagnosis of LV 
aneurism thrombosis compared to CVG. On the other hand, results of CVG significantly correlated with both MSCT and EchoCG results data. Despite the fact that CVG is considered to be an invasive "golden standard" in diagnosing LV structural pathology, high accuracy of MSCT and EchoCG is explained due to visualization of the whole LV wall with characteristic myocardial signal. During CVG invasive cardiologist sees only endocardial contour outlined by contrast, which might be a cause of false positive or false negative diagnosis, especially in case of a small aneurism, which is shown by our study data by intraoperational findings of LV aneurism and its thrombosis and corresponds to reference data (Budoff, 2004; Dirksen et al., 2002; Dolzhenko et al., 2007; Masaki et al., 2005; Schuijf et al., 2004). Besides, during MSCT or EchoCG thrombotic tissue has certain structural texture, significantly visually different from one of myocardium, which explains higher accuracy of these methods in aneurism thrombosis diagnosis compared to CVG, especially in case of flat and thin parietal clot (Laissy et al., 2007), when CVG does not show significant contrast filling defect. These considerations are confirmed by results of our study and may explain high accuracy, sensitivity and positive predictive value of MSCT in LV aneurism thrombosis diagnosis. MSCT and EchoCG show identical positive and negative predictive value in LV aneurism thrombosis diagnosis, while EchoCG shows significantly higher specificity and positive predictive value in LV chronic aneurism diagnosis compared to MSCT.

\subsection{Study limitations}

In case of defining LV volumes and EF EchoCG is the method of preference being reliable, non-invasive and, thus, having minimum of limitations (Becher et al., 2004; Budoff, 2004; Dolzhenko et al., 2007; Swedberg et al., 2005). Despite good correlations between all methods of visualization, MSCT seemed to give seriously lower absolute values of LV EF compared to EchoCG. Higher accuracy in defining LV global contractility by EchoCG may be explained by the fact, that LV volumes quantification by EchoCG is performed in two perpendicular planes under visual and manual control of sonographist. On the other hand, in CVG or MSCT EDV and ESV quantification is performed automatically by installed software in one fixed projection, which may lead to inaccuracy, especially in case of marked LV eccentric remodeling and aneurism presence (Budoff, 2004; Dirksen et al., 2002; Fleisher et al., 2007; Scanlon et al., 1999).

\section{Conclusion}

In the patients with LV chronic postinfarction aneurism data of non-invasive methods highly correlate with CVG data. In LV chronic aneurism diagnosis transthoracic EchoCG and MSCT have high accuracy compared to CVG due to high sensitivity and positive predictive value of these methods. There was no significant difference between prognostic value of MSCT and transthoracic EchoCG in LV chronic aneurism and its thrombosis diagnosis, which allows to consider MSCT a reliable alternative to EchoCG in LV structural anomalies diagnosis in the patients after AMI prior to planned CABG+AE. Data provided by MSCT and transthoracic EchoCG in LV chronic aneurism and its thrombosis significantly highly correlate with "golden standard" CVG results, which allows to consider these non-invasive methods highly reliable in defining the discussed myocardial structural anomalies. 


\section{Acknowledgment}

Authors thank Prof. Anatoliy Rudenko, Head of Coronary Heart Disease Surgeon Treatment Department of The N.M.Amosov Institute Of Cardiovascular Surgery Of AMS Of Ukraine, and Dr. Oleg Sharayevskiy, Head of MSCT Department of NSC “The N.D.Strazhesko Institute Of Cardiology" Of AMS Of Ukraine, for the provided CCA and MSCT results data for the studied patients.

\section{References}

Becher $\mathrm{H}$. et al. BSE procedure guidelines for the clinical application of stress echocardiography, recommendations for performance and interpretation of stress echocardiography: A report of the British Society of Echocardiography Policy Committee. Heart, Vol. 90 (December 2004), pp. vi23 - vi30.

Budoff M.J. Tracking Progression of Heart Disease with Cardiac Computed Tomography. Journal of Cardiovascular Pharmacology and Therapeutics, Vol. 9 (April 2004), pp. 75 82.

Dirksen M.S., et al. Usefulness of dynamic multislice computed tomography of left ventricular function in unstable angina pectoris and comparison with echocardiography. Am. J. Cardiol, Vol. 90 (February 2002), pp. 1157-1160.

Dolzhenko M.N. et al. Left ventricle diastolic function in the patients after coronary arteries bypass graft combined with left ventricle aneurismectomy according to tissue doppler imaging: one year follow-up. Postgrad. Med. J, Vol. 83 (May 2007), pp. 320324.

Dolzhenko M.N. et al. Changes in left ventricle myocardial function in patients with postinfarction cardiosclerosis according to tissue Doppler imaging after coronary arteries bypass graft combined with left ventricle aneurismectomy. Heart and vessels, No. 2 (Aptil 2008), pp. 34-41.

Fleisher Lee A. et al. ACC/AHA 2007 Guidelines on Perioperative Cardiovascular Evaluation and Care for Noncardiac Surgery: A Report of the American College of Cardiology/American Heart Association Task Force on Practice Guidelines (Writing Committee to Revise the 2002 Guidelines on Perioperative Cardiovascular Evaluation for Noncardiac Surgery. Circulation, Vol. 116 (October 2007), pp. e418 e500.

Fleming R.M. A Tate-en-Tate Comparison of Ejection Fraction and Regional Wall Motion Abnormalities as Measured by Echocardiography and Gated Sestamibi SPECT. Angiology, Vol. 53, 3 (May 2002), pp. 313-321.

Fleming R.M., Boyd L.D. High-Dose Dipyridamole and Gated Sestamibi SPECT Imaging Provide Diagnositic Resting and Stress Ejection Fractions Useful for Predicting the Extent of Coronary Artery Disease. Angiology, 53, 4 (July 2002), pp. 415-421.

Kasner M. et al. Utility of Doppler Echocardiography and Tissue Doppler Imaging in the Estimation of Diastolic Function in Heart Failure With Normal Ejection Fraction: A Comparative Doppler-Conductance Catheterization Study. Circulation, Vol. 116 (August 2007), pp. 637 - 647.

Kyung-Jong Yoo, et al. The comparison of the graft patency after coronary artery bypass grafting using coronary angiography and multi-slice computed tomography. Eur. J. Cardiothorac. Surg., Vol. 24 (July 2003), pp. 86. 
Laissy Jean-Pierre et al. Comprehensive evaluation of preoperative patients with aortic valve stenosis: usefulness of cardiac multidetector computed tomography. Heart, Vol. 93 (September 2007), pp. 1121 - 1125.

Masaki Y. et al. Cardiac Functional Analysis with Multi-Detector Row CT and Segmental Reconstruction Algorithm: Comparison with Echocardiography, SPECT, and MR Imaging. Radiology, Vol. 234 (October 2005), pp. 381 - 390.

Mok C.K., Cheung K.L., WangR.Y. Unruptured right coronary sinus to left ventricle aneurysm diagnosed by cross sectional echocardiography. Heart, Vol. 53 (February 1985), pp. $226-229$.

Petry A., Sabin K. (2003). Demonstrable statistics in medicine, GAOTAR-MED, Moscow

Rebrova O.Y. (2002). Statistical analysis of medical data, Media Sphere, Moscow

Scanlon P.J. et al. ACC/AHA Guidelines for Coronary Angiography: Executive Summary and Recommendations. Circulation, Vol. 99 (May 1999), pp. 2345-57.

Schuijf J.D. et al. Noninvasive Angiography and Assessment of Left Ventricular Function Using Multislice Computed Tomography in Patients With Type 2 Diabetes. Diabetes Care, Vol. 27 (December 2004), pp. 2905 - 2910.

Swedberg K. et al. Guidelines for Diagnosis and Treatment of Chronic Heart Failure. Eur. Heart Journ., Vol. 26 (June 2005), pp. 1115-1140. 


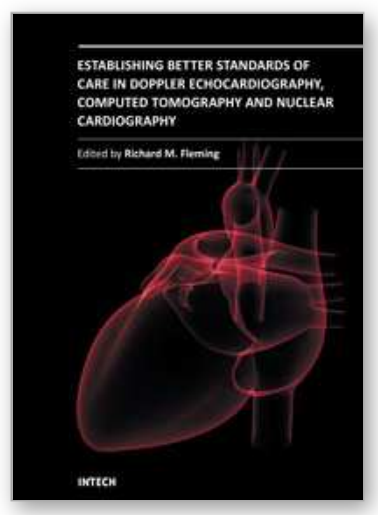

\author{
Establishing Better Standards of Care in Doppler \\ Echocardiography, Computed Tomography and Nuclear \\ Cardiology \\ Edited by Dr. Richard M. Fleming
}

ISBN 978-953-307-366-8

Hard cover, 260 pages

Publisher InTech

Published online 13, July, 2011

Published in print edition July, 2011

Since the introduction of Doppler Echocardiography, Nuclear Cardiology and Coronary CT imaging, clinicians and researchers have been searching for ways to improve their use of these important tools in both the diagnosis and treatment of heart disease. To keep up with cutting edge improvements in these fields, experts from around the world have come together in this book to provide the reader with the most up to date information to explain how, why and when these different non-invasive imaging tools should be used. This book will not only serve its reader well today but well into the future.

\title{
How to reference
}

In order to correctly reference this scholarly work, feel free to copy and paste the following:

Maryna N. Dolzhenko, Sergey V. Potashev and Natalia N. Nosenko (2011). Left Ventricle Postinfarction Aneurism: Comparison Between Diagnostic Value of Different Methods of Visualization, Establishing Better Standards of Care in Doppler Echocardiography, Computed Tomography and Nuclear Cardiology, Dr. Richard M. Fleming (Ed.), ISBN: 978-953-307-366-8, InTech, Available from:

http://www.intechopen.com/books/establishing-better-standards-of-care-in-doppler-echocardiographycomputed-tomography-and-nuclear-cardiology/left-ventricle-postinfarction-aneurism-comparison-betweendiagnostic-value-of-different-methods-of- $v$

\section{INTECH}

open science | open minds

\author{
InTech Europe \\ University Campus STeP Ri \\ Slavka Krautzeka 83/A \\ 51000 Rijeka, Croatia \\ Phone: +385 (51) 770447 \\ Fax: +385 (51) 686166 \\ www.intechopen.com
}

\author{
InTech China \\ Unit 405, Office Block, Hotel Equatorial Shanghai \\ No.65, Yan An Road (West), Shanghai, 200040, China \\ 中国上海市延安西路65号上海国际贵都大饭店办公楼 405 单元 \\ Phone: +86-21-62489820 \\ Fax: +86-21-62489821
}


(C) 2011 The Author(s). Licensee IntechOpen. This chapter is distributed under the terms of the Creative Commons Attribution-NonCommercialShareAlike-3.0 License, which permits use, distribution and reproduction for non-commercial purposes, provided the original is properly cited and derivative works building on this content are distributed under the same license. 